\title{
Tornar-se pai/mãe de uma criança com transtornos graves do desenvolvimento
}

\section{Becoming father/mother of a child with serious problems of development}

\author{
Vitor Franco ${ }^{1}$
}

\begin{abstract}
RESUMO
O interesse pela pesquisa sobre os pais das crianças com transtornos graves do desenvolvimento tem conhecido diferentes focos de interesse ao longo do tempo, desde uma abordagem mais psicopatológica, interessada em descrever os aspectos negativos gerados pelo impacto de ter um filho com deficiência, passando pelo estudo das estratégias de coping usadas para lidar com a situação, até ao conhecimento das forças e da resiliência que esses pais mobilizam nas suas vivências. A grande mudança, no entanto, deriva de os pais/mães serem considerados não apenas em função da criança, mas também pelo que acontece no seu próprio processo de desenvolvimento. Esta perspectiva desenvolvimental é aqui abordada a partir da construção dos vínculos fundamentais presentes na ligação entre mãe/pai e o seu bebé, o modo como tal ligação é quebrada e como pode ser reconstruída quando a criança apresenta um diagnóstico de transtorno grave. Só a retoma do seu próprio processo de desenvolvimento, por parte dos pais, lhes permitirá exercer plenamente a sua parentalidade de forma emocionalmente adequada e consistente, assente numa responsividade permanente perante as circunstâncias da vida quotidiana. Esta perspectiva permite abrir novos desafios interdisciplinares quanto à forma de trabalhar na intervenção precoce no desenvolvimento infantil e a uma leitura abrangente do trabalho centrado na família.
\end{abstract}

Palavras-chave: deficiência; intervenção precoce; adaptação familiar; transtorno de desenvolvimento; parentalidade.

DOI: $10.1590 / 0104-4060.44689$

1 Universidade de Évora. Évora, Portugal. Departamento de Psicologia, Apartado 94, 7000-000.E-mail: vfranco@uevora.pt 


\begin{abstract}
The interest in research on parents of children with severe developmental disorders has known different focuses of interest over time, from a more psychopathological approach, interested in describing the negative aspects of the impact of having a child with disabilities, to the study of coping strategies used to deal with the situation, and the study of the strength and resilience mobilized by these parents. The big change, however, is that the concern about parents is not only because of the child, but also for what happens in their own development processes. This developmental perspective is addressed here to the construction of the fundamental attachment between mother/father and the baby, how this bond is broken, and how it can be reconstructed when the child has a severe disability diagnosis. Only the resumption of parents' developmental process will enable them to perform their parenting in an emotionally appropriate and consistent manner, based on adequate responsiveness in everyday life situations. This view allows to open new interdisciplinary challenges about how to work on early intervention in child development and to a comprehensive understanding of the family centered intervention.
\end{abstract}

Keywords: disabilities; early intervention; family adaptation; developmental disorder; parenthood.

\title{
Introdução
}

O nascimento de uma criança com deficiência ou transtorno grave de desenvolvimento é um acontecimento que, embora sempre temido, nunca é esperado e tem um impacto emocional significativo sobre a família e cada uma das pessoas que a compõem, que veem assim o seu desenvolvimento também profundamente afectado.

A pesquisa acerca dos pais e mães dessas crianças (os estudos com mães são consideravelmente mais frequentes) tem tido alterações significativas ao nível do seu foco de interesse, ao longo do tempo, e podemos considerar quatro enfoques principais. (KNIGHT, 2013).

Uma perspectiva marcadamente psicopatológica marcou os primeiros estudos (VON GONTARD et al., 2002) especialmente enfocados na depressão, estresse, sobrecarga e isolamento social desses pais/mães, sempre numa perspectiva do seu disfuncionamento psicológico. Ser pai/mãe de uma criança com deficiência era assumido como algo fundamentalmente negativo e perturbador, com impacto pernicioso na vida, dependendo da severidade de cada caso, as 
características da criança e a sobrecarga que ela representa para a família. Uma leitura mais psicodinâmica privilegiava a relevância da ferida narcísica gerada pela deficiência e um quadro essencialmente depressivo, ou de depressividade crónica, enquanto num enfoque mais comportamental foram mais valorizados os aspectos descritivos da situação emocional dos pais e o modo como a sua vida social é afectada.

Nas últimas décadas do século passado prevaleceram os modelos de estresse e coping especialmente interessados no estudo dos factores de estresse e das necessidades das famílias. (JOHNSTON et al., 2003; LEWIS et al., 2006). Com eles foram valorizados os instrumentos para avaliação dos problemas parentais e especialmente os processos de tomada de decisão. A diferença entre um bom e um mau processo de adaptação à deficiência foi colocada, sobretudo, ao nível das estratégias (cognitivas e pragmáticas) para lidar com a situação.

Num terceiro momento a situação deixou de ser entendida como necessariamente negativa e perturbadora e o interesse dos pesquisadores centrou-se nos modelos de resiliência, colocando o foco da pesquisa nas forças e fortalezas dos pais. (GREEFF; NOLTING, 2013; FEENEY, 2000). O foco deslocou-se, assim, dos aspectos negativos de ter um filho com deficiência, para o interesse em conhecer os factores positivos, em particular os factores de proteção, suporte social e resiliência, que permitem que os pais e mães respondam adequadamente às necessidades das suas crianças, mantendo a organização e coesão familiar e níveis de bem-estar emocional e social adequados.

Mais recentemente um novo paradigma da pesquisa sobre os pais das crianças com deficiência tem-se preocupado, sobretudo, em compreender os processos de adaptação e transformação que ocorrem nos pais e na família. (KNIGHT, 2013). Considerando tanto as dimensões internas como as de suporte, os aspectos positivos e os constrangimentos, sendo que a situação deixa de ser vista como intrinsecamente negativa, para ser vista enquanto um processo real e significativo de transformação. (TRUTE et al., 2010; RIEGER; MCGRAIL, 2013; TRUTE et al., 2012, DEMPSEY et al., 2009).

Todos os diferentes modelos teóricos têm procurado descrever esse processo de adaptação à deficiência, visando compreender a diversidade de respostas encontradas nessas famílias ao nível das suas vivências e da forma como se relacionam com a criança. (MONTEIRO et al., 2002). Apesar da diversidade de enfoques e perspectivas, há em todos alguns aspectos comuns, admitindo que a adaptação é um processo complexo e multifacetado. (KNIGHT, 2013; BAILEY et al., 2008; EMERSON, 2003). Dos mais antigos aos mais recentes, todos eles enfatizam a importância dessa multidimensionalidade de factores reconhecendo a importância de variáveis relativas aos pais/mães (suas características, personalidade e estilos cognitivos), variáveis da criança, incluindo a natureza do 
transtorno ou déficit e da sua funcionalidade, e variáveis relacionadas com os contextos de vida e de participação ao nível social.

Embora todos esses modelos tentem integrar e descrever aquilo que acontece, falham frequentemente na sua dimensão desenvolvimental, em parte pela falta de estudos longitudinais que a possam esclarecer. Mas também porque esse processo de adaptação deve considerar não apenas o desenvolvimento dos pais/mães enquanto processo global, como também o lugar que a parentalidade (desde a gravidez a tudo o que acontece antes, no decurso e na sequência do diagnóstico) desempenha nesse processo de desenvolvimento.

Optamos, neste texto, por nos referirmos a pai/mãe porquanto pretendemos abordar aquilo que acontece em particular com cada um dos progenitores da criança com transtornos graves do desenvolvimento. Enquanto falar da adaptação familiar nos remete para uma abordagem mais sistémica do conjunto da família, aqui queremos colocar o foco no modo diferenciado como o pai e a mãe fazem o seu processo pessoal de desenvolvimento, muitas vezes em ritmos e percursos bem diversos e a que não serão até alheios os papéis e estereótipos relacionados com as diferenças de género.

Quando é feito o diagnóstico de uma patologia severa, seja na altura do nascimento seja em qualquer outro momento ao longo da infância, há inevitavelmente uma crise. As dimensões emocionais consideradas mais frequentemente na pesquisa com pais/mães (estresse, raiva, negação ou depressão) (MCCARTHY et al., 2006) podem ser consideradas como as primeiras respostas emocionais à perda da idealização daquela criança que eles tinham construído. A idealização, ou seja, a construção de uma representação do bebé ideal, está precisamente no início da constituição da parentalidade. Ela acompanha a gravidez, ao longo da qual a imagem de um bebé perfeito, desejado, bonito e do qual tudo se pode esperar, vai sendo construída. Essa idealização tem também uma função fundamental que é permitir cuidar do bebé real e o desenvolvimento do apego.

Daí que o fim dessa idealização, e com ela a inevitável perda de esperanças e expectativas, seja sempre um processo doloroso de luto, em que os sentimentos depressivos são inevitáveis e, por isso, estão sempre presentes na pesquisa, como uma reação imediata ao diagnóstico ou ao longo do tempo. $\mathrm{O}$ problema central passa a ser como é que esses pais podem alcançar um novo nível de funcionamento depois da crise, apesar do seu sofrimento, tornando-se pais/mães amorosos e nutrientes, e não apenas funcionais e utilitários, para um filho que não corresponde à sua idealização.

$\mathrm{Na}$ avaliação do impacto das práticas de intervenção precoce no desenvolvimento infantil, numa perspectiva centrada na família, tem-se verificado que melhorar o funcionamento familiar e aumentar o suporte social são os objectivos mais difíceis de alcançar. (FRANCO; APOLÓNIO, 2008; FRANCO; MELO; 
APOLÓNIO, 2012). Por seu lado, alguns programas tendem a ignorar o que acontece nos desenvolvimento dos pais ou apenas se preocupam em providenciar suporte, instrumentos ou competências para que executem as suas funções, sem cuidar de verificar se eles estão em condições psicológicas e emocionais de o fazerem de modo relacionalmente saudável. Por isso, nos parece fundamental compreender como o processo desenvolvimental dos pais/mães está relacionado com mudanças emocionais em ordem a promover a resiliência e o apego.

\section{Os pais e mães face ao diagnóstico da deficiência}

A causa ou etiologia dos transtornos do desenvolvimento é muito variável e até, em muitos deles, desconhecida. A detecção de sinais que permitam chegar bem cedo a um diagnóstico é, no entanto, fundamental, porque só isso permite fazer intervenção precoce, ou seja, agir cedo no processo de desenvolvimento. Daí que desde muito cedo os pais estejam envolvidos tanto na deteç̧ão desses indicadores como na procura de compreensão e diagnóstico.

Em estudo sobre o impacto do processo de diagnóstico (ou seja, como os pais sentiram e o que pensaram face ao diagnóstico que lhes foi comunicado, neste caso concreto a Síndrome de X Frágil) encontramos vários aspectos emocionais:

- Esperança ilusória no rótulo. Como se o encontrar a etiologia e dar o nome à Síndrome fosse o mesmo que encontrar uma solução. Ou quando isso parece, momentaneamente, afastar medos maiores, e.g. a mãe que pensa, aliviada, "afinal não é autismo"; Eu toda descansada, porreiro,... como se o rótulo fosse a solução

- Desilusão e frustração. Há pais e mães que referem que nessa primeira confrontação com o diagnóstico a primeira coisa que pensaram foi que não poderiam mais ser felizes;

- Destrutividade. Como se o diagnóstico, não esperado, fosse uma sentença para a vida, uma condenação.

- Interrogação e confusão. Um turbilhão de interrogações e dúvidas, muitas delas sem resposta: o que se passa? e agora? o que faço? o que é isto? por quê?

Esse impacto teve, obviamente, consequências emocionais de curto e médio prazo: sofrimento, choro, sentimento de ser único, estar só e isolado, sufoco, não conseguir dormir nem descansar. (FRANCO, 2013, p. 114). 
A partir do confronto com a notícia que é trazida pelo diagnóstico, coloca-se o problema do que fazer de seguida. Muitas vezes os transtornos de desenvolvimento são encarados como se fossem doenças, ou seja, situações anómalas e temporárias que carecem de um tratamento para ser anuladas, e pais e técnicos tendem a seguir um tipo de pensamento próprio do modelo médico, centrado na procura do que provoca a situação e de como se pode tratar. A primeira pergunta é frequentemente sobre qual o tratamento adequado para a criança.

No entanto, de um ponto de vista do desenvolvimento, a deficiência ou distúrbio grave do desenvolvimento é algo bem diferente. Porque o que está em causa é todo o desenvolvimento, em todas as áreas e ao longo de toda a vida. Para os transtornos de desenvolvimento, que são condições de carácter permanente, não há cura. Pelo contrário, por muito favorável que seja o quadro, é algo que vai marcar a vida da pessoa, em todas as áreas e dimensões e colocar desafios aos seus contextos de vida. Consequentemente, o tratamento também não é o que conduz à cura, mas tudo aquilo que leva ao máximo desenvolvimento e à máxima inclusão dessa criança.

É nesta perspectiva longitudinal que temos de situar as famílias. Na pesquisa com famílias com Síndrome de X Frágil encontramos frequentemente pais que passam anos procurando um diagnóstico, colocando na expectativa desse diagnóstico uma esperança de cura. Mas muitos nos relatam como o dia a seguir ao diagnóstico que buscaram durante tanto tempo foi de perplexidade, pela constatação óbvia que o diagnóstico nada mudava do seu filho. (FRAN$\mathrm{CO}, 2013)$. O que não quer dizer que não seja muito importante, mas os pais percebem que não é o diagnóstico que traz a cura.

A expectativa de cura (ou de tratamento) é, no entanto, compreensível. Certamente que todos os pais de crianças com transtornos graves do desenvolvimento gostariam que, quando lhes é dado o diagnóstico lhes fosse logo dito que o problema tem cura, completa, rápida e eficaz, que é fácil e está acessível. No entanto, não é assim com as deficiências, e o trabalho com famílias exige que os técnicos o tenham bem claro. Mais do que isso, deixar que a família se instale na perspectiva da cura e tratamento comporta um risco grande e triplo. Em primeiro lugar risco de exclusão da criança, em segundo lugar risco acrescido para o seu desenvolvimento e, em terceiro lugar, risco para o desenvolvimento e saúde mental dos próprios pais.

Risco de exclusão porque o desenvolvimento de uma criança é indissociável de um processo de ligação e envolvimento, ou seja, de inclusão, que começa precisamente pela inclusão familiar. Toda a criança necessita de um lugar para nascer e se desenvolver no contexto relacional de uma família (lugar que é construído a partir da idealização, como vimos anteriormente). Quando a família pensa que o trabalho principal deve ser feito por um técnico, um terapeuta, um profissional 
de ajuda médica ou educativa, está a dar um primeiro e amplo passo no sentido da exclusão da criança. Porque o processo de inclusão não começa na entrada na escola, mas sim no nascimento, e sem inclusão familiar não há inclusão educativa, e sem esta não haverá inclusão social ou profissional. Quando a família incorre na ilusão de que alguém vai tratar (fazer tratamento) do seu filho que tem um transtorno do desenvolvimento, facilmente descuida o desenvolvimento e a inclusão. Este é um dos grandes desafios epistemológicos dos nossos dias: mudar a abordagem dos transtornos de desenvolvimento (tanto por parte dos profissionais como das famílias) de um modelo médico tradicional, centrado no problema, para um modelo centrado na família e no desenvolvimento, tendo em conta tudo o que sabemos hoje acerca de como este se processa e se promove.

Trata-se também de um risco acrescido para o desenvolvimento da criança. Embora a antiga, e ainda muitas vezes usada, designação de risco estabelecido seja paradoxal na língua portuguesa, porque, por um lado "risco" se refere a uma possibilidade ou probabilidade de algo poder acontecer e, por outro, "estabelecido" remete para aquilo que é determinado, já existente e estável. Esta terminologia, proposta por Tjossem (1976) organizando o risco em três categorias (estabelecido, biológico e ambiental) afigura-se hoje pouco rigorosa para descrever e abordar quer os factores de risco quer a forma como o que já existe, seja uma síndrome ou um transtorno de desenvolvimento, afecta o futuro da criança ou a pode colocar em perigo. No entanto, um transtorno do desenvolvimento comporta riscos e perigos de diferente natureza e essas crianças passam a estar em risco também ambiental e social. Pois o mais importante para o seu crescimento e desenvolvimento (já que, obviamente, não deixam de estar em desenvolvimento por terem um problema) são as interações que estabelecem com os que lhe são próximos e nos contextos concretos da sua vida. Se os pais não estão disponíveis, emocionalmente, ou se afastam para as entregar a alguém que, supostamente, vai resolver a situação, estão a colocar em causa o desenvolvimento. A primeira coisa de que a criança com deficiência necessita, tanto quanto qualquer outra criança, é de pais, pai e mãe, que sejam cuidadores e amorosos. Claro que ser pai/mãe de uma criança assim (com eventuais limitações na sua capacidade para interagir, responder ou tomar iniciativas) é ainda mais difícil do que ser pai/mãe de qualquer outra criança sem essas limitações. Mas pais que entregam o desenvolvimento dos seus filhos a outrem os colocam em risco na medida em que poderão não lhes estar a dar aquilo que é mais importante: disponibilidade, interacção, envolvimento, transação, sincronia.

Os pais continuam a ser a principal influência no desenvolvimento dos seus filhos, mesmo quando essas crianças beneficiam de programas de 
intervenção, e a eficácia da intervenção está altamente relacionada com a eficácia dos pais em manifestarem esse processo interativo que se demonstrou influenciar o desenvolvimento das crianças que não estão envolvidas em programas de intervenção. (MAHONEY; PERALES, 2013, p. 212).

O conceito de estimulação precoce é uma ideia antiga, limitada e ultrapassada que considerava a criança como basicamente passiva e receptora, e que os outros, especialmente os técnicos, iriam promover o seu desenvolvimento agindo sobre ela. Sabemos hoje que não é assim que acontece o desenvolvimento infantil. A criança não é passiva e não é a ação pontual sobre ela que tudo resolve, mas é num continuum de interações, relações e transações que o desenvolvimento acontece, vinte quatro horas por dia. É por isso que a consideração do papel da família é imprescindível na compreensão do desenvolvimento da criança com transtornos graves.

Os pais também estão em risco, porque aqueles pais que se limitam a entregar os seus filhos para tratamentos (sejam farmacológicos, reabilitativos ou educativos) e descuram o seu papel e envolvimento parental estão, também, a dar sinais de que algo no seu desenvolvimento está ameaçado ou em perigo. Porque ter um filho com um transtorno grave do desenvolvimento é um desafio enorme ao funcionamento emocional, afectivo e social de qualquer pai/mãe e, consequentemente, à sua saúde mental e bem-estar. Um filho ocupa um lugar particular no processo de desenvolvimento daquele que vai ser pai/mãe. E tal como o filho necessita dos pais para se desenvolver, também o pai/mãe só pode ser cuidador adequado para o seu filho se ele mesmo estiver em processo de desenvolvimento. Ou seja, se não se atolou nesse processo, no pântano da sua dor e confusão, se não teve de cortar esse filho do seu pensamento e do seu coração. (OLSSON; HWANG, 2001). Ninguém pode ser pai/mãe de uma criança com transtorno grave do desenvolvimento apenas por obrigação ou dever moral. Tais podem ser pais funcionais ou utilitários, cumpridores e até dispostos a fazer tudo aquilo que os médicos, psicólogos, terapeutas ou professores lhes exijam ou sugiram. Mas não é disso que a criança necessita, nem o que se espera de um pai/mãe. Um filho é parte de si mesmo, de um sonho, de um desejo, de um projecto de vida, uma parte do seu Self. Pais que vivem na negação das dificuldades do seu filho não podem ajudar a que ele se desenvolva. Pais prisioneiros da sua raiva e revolta, ou da culpa, não podem cuidar dos seus filhos, tentam cuidar apenas de si mesmos. Do mesmo modo que pais/ mãe imersos no sofrimento depressivo e no desejo de voltar ao filho perfeito que começaram por desejar (e que existiu até ao momento do diagnóstico) não podem ajudar os seus filhos a crescer. 


\section{A intervenção precoce centrada na família e o tornar-se pai/mãe}

Depois dos primeiros momentos de impacto da notícia do diagnóstico e do posicionamento dos pais perante a intervenção, a mobilização para um programa de intervenção precoce é então fundamental. A eficácia dos programas de intervenção precoce no desenvolvimento infantil está directamente dependente da forma de envolvimento das famílias.

Mahoney e Perales (2013) chamam a atenção para que nos programas de intervenção que se limitam a oferecer serviços terapêuticos, sob a forma de respostas no domínio da saúde, educação ou proteção social, sem envolvimento dos pais e em que a ênfase está nas competências técnicas e na qualidade dos serviços disponibilizados, os resultados obtidos são em geral muito baixos. Esses programas são construídos a partir de uma perspectiva tradicional centrada na problemática e na criança e que desvaloriza o tempo que os pais passam com os seus filhos, que é significativamente mais do que o dos serviços terapêuticos ou educativos.

Já nos programas que promovem a participação e envolvimento dos pais e em que estes participam activamente na intervenção os resultados são mais evidentes. Daí o sucesso de toda uma nova geração de programas centrados na família, em que esta é chamada a trabalhar em parceria com os profissionais e a estender a intervençãoo para além dos limites restritos da intervenção terapêutica ou de reabilitação.

No entanto, Mahoney e Perales esclarecem que dentro do grupo de programas de intervenção precoce há dois tipos bem diferenciados na sua concepção e resultados. Num deles, quando os pais são chamados a intervir para desempenhar papéis que anteriormente eram assegurados pelos técnicos e a sua função passa a ser estimular, ensinar e tratar, os resultados não se diferenciam dos do primeiro grupo e são igualmente baixos ou nulos.

Resultados significativamente diferentes verificam-se num outro tipo de programa em que os pais participam ativamente, mas em que o que lhes é solicitado é que sejam mais efetivos na sua parentalidade, ou seja, que se tornem pais mais responsivos em relação aos seus filhos, como fariam com outro filho que não apresentasse transtornos do desenvolvimento. Dizem os autores:

Os pais desempenham um papel fundamental na promoção do desenvolvimento precoce dos seus filhos; e a responsividade parental é uma das principais qualidades parentais, promotora de aprendizagem e desenvolvimento da criança. Os estudos indicam que a intervenção não foi eficaz 
a não ser quando promoveu a responsividade parental para com os seus filhos, e que o desenvolvimento das crianças pode ser promovido através do encorajamento dos pais a interagirem de forma mais responsiva com os seus filhos. (MAHONEY; PERALES, 2013, p. 219).

Vemos aqui uma clara correspondência com três momentos no processo de adaptação (FRANCO, 2009) e de se tornar pai/mãe.

No primeiro grupo de programas, a procura de estimulação sem envolvimento dos pais, liga-se, fundamentalmente, com as diferentes formas de negação, quer como causa quer como consequência. Ou porque os pais procuram esses programas em consequência da sua negação, buscando a cura num movimento de retoma da idealização inicial e de retorno ilusório a ela. Ou então porque a natureza dos programas coloca intencionalmente os pais de fora e não lhes possibilita uma vivência plena da sua parentalidade, como se a família não existisse enquanto contexto social e relacional primordial em todo o desenvolvimento da criança. O que promove igualmente a negação, tanto da problemática como do reconhecimento, por parte dos pais, do seu papel e das suas competências parentais e relacionais.

O segundo grupo de programas também corresponde a um outro momento do desenvolvimento dos pais e mães. O processo de adaptação não fica terminado no luto, embora aí termine muita da literatura mais patologizante sobre os processos adaptativos. Se o processo de adaptação terminasse no luto, então estes pais estariam sempre perante o risco de dor crónica (como também há quem defenda) perante algo que sempre estará em falta. No caso do luto já elaborado teríamos os pais funcionais e utilitários, os que se dispõem a fazer pela criança aquilo que é pedido pelas equipes e técnicos. Os programas de intervenção precoce que, mesmo dizendo-se centrados na família, exigem dos pais procedimentos de tipo técnico (transferindo para eles o que tradicionalmente era saber técnico) contentam-se com a não negação, ou aquilo que muita vezes é significado pelo aceitar. Aceitar como sendo o assumir passivo de uma inevitabilidade, a partir da qual se define um conjunto de papéis parentais: alimentar, transportar, vestir, estimular. Também aqui temos pais em risco de estagnação no seu desenvolvimento, ou pelo menos nalgumas áreas ou domínios desse desenvolvimento. A mobilização dos pais para exercerem tarefas terapêuticas é, por vezes, feita através da culpabilidade que os coloca numa situação de fragilidade face às propostas de acção dos serviços que oferecem os programas, e comporta riscos conhecidos para a saúde mental e bem-estar emocional.

Em relação ao terceiro tipo de programas, em que Mahoney e Perales (2013) enfatizam a responsividade, dentro de uma perspectiva de parentalidade, 
eles implicam um processo de desenvolvimento dos pais que é de outra natureza. Exige que não tenha parado no luto pela idealização inicial (e respectiva elaboração), mas que se construa do lado da relação. O que representa a reconstrução de um vínculo.

A possibilidade de ser responsivo, estar atento e responder de modo adequado às solicitações, é um processo fundamental. Aliás, corresponde ao que se espera que sejam os comportamentos básicos de todos os pais. Brazelton e Cramer (1989) descrevem precisamente cinco características nas condutas parentais no desenvolvimento fundamental de cada bebé: sincronia, simetria, contingência, encadeamento/adesão, jogos/brincadeiras e autonomia e flexibilidade. A responsividade corresponde então a essa acção permanente e quotidiana dos pais/mães disponíveis para responder às solicitações da criança. Mais do que às necessidades, já que as solicitações nos dão a ideia de uma criança activa $\mathrm{e}$ que procura no outro e no seu contexto aquilo de que necessita para se desenvolver. Os pais/mães para poderem ter esse papel fundamental devem ter podido retomar o seu desenvolvimento. No qual o desejo de ter um filho foi certamente importante e estruturante e agora o desejo daquele filho é imprescindível.

O modelo de parentalidade enfatiza as práticas de intervenção que maximizam o uso, pelos pais, das qualidades interactivas que a pesquisa tem mostrado estarem associadas com o desenvolvimento infantil. A pesquisa indica que a responsividade parental representa uma influência decisiva no desenvolvimento e bem-estar sócio-emocional das crianças com Síndrome de Down ou outras deficiências. (MAHONEY; PERALES, 2013, p. 220).

Não admira que muitas vezes pais e mães, ao sentirem a importância desse desejo, o façam sobre negação de outras dimensões da sua vida, por exemplo, quando consideram aquele filho como "um anjo" na sua vida ou como "a melhor coisa que lhes aconteceu na vida". Não que a presença da criança com deficiência não possa ser vista de forma positiva. Aliás, terá sempre de o ser, mas no reconhecimento inicial das suas condições e realidade, para, a partir daí construir novas idealizações.

$\mathrm{Na}$ idealização se cria a força para as tarefas impossíveis e desafiantes no cuidar de um bebé (ou de um filho em geral, ou de um filho com deficiência). A criança real, com uma deficiência e bem diferente da que tinha sido imaginada, tem de ser igualmente ser idealizada pelos seus pais como condição para um real apego e um ajustamento adequado. 
Uma nova idealização terá de ter todas as componentes da original (da pré-história do apego), nas palavras de Brazelton e Cramer (1989), nomeadamente beleza, competência e esperança.

\section{Conclusão}

Poder ajudar uma mãe ou um pai no seu processo de adaptação e desenvolvimento face à deficiência do seu filho exige, da parte dos profissionais, uma compreensão (necessariamente empática) dos processos internos e emocionais envolvidos no desejo de ser mãe/pai, tanto quanto das condições sociais e de vida quotidiana dessas famílias.

Se os programas de intervenção precoce descurarem os aspectos emocionais envolvidos no processo de desenvolvimento da parentalidade, centrando-se apenas nas competências pragmáticas e funcionais, correm o risco de colocarem sobre os pais/mães e família um fardo acrescido, aumentando a negação (com esperança ilusória de tratamento e cura) ou a culpa (com o peso da vergonha e da obrigação).

Só o trabalho sobre o significado do tornar-se pai/mãe de uma criança com um transtorno do desenvolvimento poderá impactar no movimento interno que cada um dos progenitores (e até de outros membros da família) inevitavelmente terá de fazer, de modo a poder construir uma parentalidade saudável (para si mesmos e para a criança).

\section{REFERÊNCIAS}

BAILEY, D. B.; SIDERIS, J.; ROBERTS, J.; HATTON, D. Child and Genetic Variables Associated With Maternal Adaptation to Fragile X Syndrome: A Multidimensional Analysis. American Journal of Medical Genetics, Part A 146A, p. 720-7299, 2008.

BRAZELTON, T.; CRAMER, B. The Earliest Relationship: Parents, Infants, And The Drama Of Early Attachment. Reading, Mass.: Addison-Wesley, 1989.

DEMPSEY, I.; KEE, D.; PENNELL, D.; O'REILLY, J.; NELIANDS, J. Parenting stress, parenting competence and family-centered support to young children with intellectual and developmental disability. Research in Developmental Disabilities, v. 30, n. 3, p. 558-566, 2009. 
EMERSON, E. Mothers of children and adolescents with intellectual disability: social and economic situation, mental health status, and the self-assessed social and psychological impact of child's difficulties. Journal of Intellectual Disability Research, v. 47, p. 385-399, 2003.

FEENEY, S. Implications of attachment style for paterns of health and illness. Child: Care, Health and development, v. 26, n. 4, p. 277-288, 2000.

FRANCO, V. A adaptação das famílias de crianças com perturbações graves do desenvolvimento - contribuição para um modelo conceptual. Infad-International Journal of Developmental and Educational Psychology, v. XXI, n. 2, 1, p. 25-37, 2009.

FRANCO, V. (Org.). Síndrome de X Frágil: pessoas, contextos e percursos. Évora: Ed. Aloendro, 2013.

FRANCO, V.; APOLÓNIO, A. M. Avaliação do Impacto da Intervenção Precoce no Alentejo: Criança, Família e Comunidade. Évora: Ed. ARS, 2008.

FRANCO, V.; MELO, M.; APOLÓNIO, A. Problemas do Desenvolvimento Infantil e Intervenção Precoce. Educar em Revista, Curitiba: Editora UFPR, n. 43, p. 49-64, jan./ mar. 2012.

GREEFF, A. P.; NOLTING, C. Resilience in Families of Children With Developmental Disabilities. Families, Systems, \& Health, v. 31, n. 4, p. 396-405, 2013.

JOHNSTON, C.; HESSL, D.; BLASEY, C.; ELIEZ, S.; ERBA, H.; DYER-FRIEDMAN, J., GLASSER, B.; REISS, A. Factors associated with parenting stress in mother of children with fragile X syndrome. Journal of Developmental and Behavioral Pediatrics, $\mathrm{v}$. 24, p. 267-275, 2003.

KNIGHT, K. The changing face of the 'good mother': trends in research into families with a child with intellectual disability, and some concerns. Disability and Society, v. 28, n. 5, p. 660-673, 2013.

LEWIS, P.; ABBEDUTO, L.; MURPHY, M.; RICHMOND, E.; GILES, N.; BRUNO, L.; SCHROEDER, S.; ANDERSON, J.; ORSMOND, G. Psychological well-being of mothers of youth with fragile X syndrome: Syndrome specificity and within-syndrome variability. J Intellect Disabil Res, v. 50, p. 894-904, 2006.

MAHONEY, G.; PERALES, F. The role of parents of children with Down syndrome and other disabilities in early intervention. In: RONDAL, J.; PERERA, J.; SPIKER, D. (Ed.). Neurocognitive Rehabilitation of Down Syndrome-Early Years. Cambridge University Press, 2013. p. 205-223.

MCCARTHY, A.; CUSKELLY, M.; VAN KRAAYENOORD, C. E.; COHEN, J. Predictors of stress in mothers and fathers of children with fragile $\mathrm{X}$ syndrome. Research in Developmental Disabilities, v. 27, p. 688-704, 2006. 
MONTEIRO, M.; MATOS, A. P.; COELHO, R. A adaptação psicológica de mães cujos filhos apresentam Paralisia Cerebral: Revisão da literatura. Revista Portuguesa de Psicossomática, v. 4, n. 2, p. 149-178, 2002.

OLSSON, M. B.; HWANG. C. P. Depression in mothers and fathers of children with intellectual disability. Journal of Intellectual Disability Research, v. 45, p. 535-543, 2001.

RIEGER, A.; MCGRAIL, J. P. Coping humor and family Functioning in parents of children with disabilities. Rehabilitation Psychology, v. 58, 1, p. 89-97, 2013.

TJOSSEM, T. D. Intervention strategies for high risk infants and young children. Baltimore: University Park Press, 1976.

TRUTE, B.; BENZIES, K. M.; WORTHINGTON, C. Mother positivity and family adjustment in households with children with a serious disability. J Child Fam Stud., v. 21, p. 411-417, 2012.

TRUTE, B.; BENZIES, K. M.; WORTHINGTON, C.; REDDON, J. R.; MOORE, M. Accentuate the positive to mitigate the negative: Mother psychological coping resources and family adjustment in childhood disability. Journal of Intellectual \& Developmental Disability, v. 35, n. 1, p. 36-43, 2010.

VON GONTARD, A.; BACKES, M.; LAUFERSWEILER-PLASS, C.; WENDLAND, C.; LEHMKUHL, K.; ZERRES, G.; RUDNIK-SCHÖNEBORN, S. Psychopathology and family stress - comparasion of boys with fragile $\mathrm{X}$ syndrome and spinal muscular atrophy. Journal of Psychology and Psychiatry, v. 43, p. 949-957, 2002.

Texto recebido em 27 de outubro de 2015 . Texto aprovado em 13 de janeiro de 2016. 